\title{
COVID-19 Pandemic, Pre-exposure Prophylaxis (PrEP) Care, and HIV/ STI Testing Among Patients Receiving Care in Three HIV Epidemic Priority States
}

\author{
Brandon J. Hill ${ }^{1,2} \cdot$ Brie Anderson $^{1} \cdot$ Li Lock $^{1}$ \\ Accepted: 9 February 2021 / Published online: 18 February 2021 \\ ( $)$ The Author(s), under exclusive licence to Springer Science+Business Media, LLC part of Springer Nature 2021
}

\begin{abstract}
The COVID-19 pandemic has the potential to disrupt HIV prevention services. We conducted an electronic health record analysis of PrEP, HIV, and STI visits at eight sexual health clinics in Arkansas, Missouri, and Oklahoma during the onset of the pandemic (March 1, 2020 to June 30, 2020) and compared the data with pre-pandemic (March 1, 2019 to June 30, 2019) volumes. Our data revealed a significant increase in the proportion of male PrEP visits during the pandemic compared to the pre-pandemic period, with the majority provided via telehealth/telePrEP. Overall, HIV and STI testing significantly decreased during the pandemic period.
\end{abstract}

Keywords HIV $\cdot$ Southern US $\cdot$ Pre-exposure prophylaxis $\cdot$ COVID-19 $\cdot$ STI testing

\section{Resumen}

La pandemia del COVID-19 tiene el potencial de interrumpir los servicios de prevención del VIH. Conducimos un análisis electrónico de expedientes de salud sobre visitas para la PrEP, el VIH, e ITS en ocho diferentes clínicas de salud sexual en Arkansas, Misuri y Oklahoma durante el inicio de la pandemia (1 de marzo de 2020 hasta 30 de junio de 2020) y comparamos esos datos con los índices previos a la pandemia (1 de marzo de 2019 hasta 30 de junio de 2019). Nuestros datos revelaron un aumento significativo en la proporción de visitas para la PrEP por personas masculinas durante la pandemia, comparado al periodo previo a la pandemia, con la mayoría de citas conducidas mediante la telemedicina/telePrEP. En general, las pruebas de VIH e ITS disminuyeron significativamente durante el periodo de la pandemia.

\section{Introduction}

The COVID-19 pandemic has the potential to negatively impact engagement with HIV prevention and care services across the United States (US) [1-3]. However, the impact may be particularly detrimental to lower resourced areas of the country, like states in the US South, which are already falling behind in meeting the Ending the HIV Epidemic: A Plan for America (EHE) goal of reducing HIV diagnoses by $90 \%$ within the next decade $[4,5]$. More specifically, seven

Brandon J. Hill

brandon.hill@ppgreatplains.org

1 Planned Parenthood Great Plains, 4401 W 109th Street Suite 200, Overland Park, KS 66211, USA

2 The Kinsey Institute, Indiana University, Bloomington, IN, USA states including those with high rates of rural HIV and STI infection: Alabama, Arkansas, Kentucky, Mississippi, Missouri, Oklahoma, and South Carolina, have been listed as high priority states in addition to 48 US counties identified as "hotspots" where more than $50 \%$ of the total new HIV diagnoses occurred from 2016 to 2017 [5]. Understanding how HIV prevention and care services in these geographical areas have been impacted by the COVID-19 pandemic is especially significant given the already strained and eroding HIV/AIDS and health care safety-net system [1].

Although the literature is limited, emerging research suggests that the COVID-19 pandemic, and subsequent state and/or local orders and restrictions, may impose both individual and structural level barriers to engaging with and accessing HIV prevention and care. For example, a recent cross-sectional survey of Australian men who have sex with men (MSM) found that 25\% of daily PrEP users stopped taking PrEP during COVID-19 pandemic shelter-in-place 
orders, with only a small proportion of users switching from daily PrEP to on-demand PrEP use [6]. In a study presented at the AIDS Conference 2020, roughly one-third of PrEP users who were under shelter-in-place orders stopped taking PrEP during the onset of the pandemic, citing low perceived HIV risk [7]. Another study, conducted at one of the largest PrEP-providing community health centers in New England (Boston, MA) found that from January to April 2020, the over 3500 PrEP-using patients had decreased by $18 \%$, PrEP initiation decreased by $72 \%$, STI and HIV testing decreased by $85 \%$, and lapses in PrEP prescription refills increased by $191 \%$, with prescription lapse rates higher among non-white PrEP users [8].

Research on the trajectories of PrEP behaviors among PrEP-users prior to and during the onset of the COVID19 pandemic highlight how changes in sexual behavior and access to care may also influence PrEP discontinuation and uptake. In a cohort study of MSM in the US South, 9\% reported they had discontinued PrEP use, with nearly a quarter reporting difficulties accessing PrEP, HIV testing, and STI testing during the onset of the pandemic [9]. Longitudinal analyses indicated significant changes in sexual behaviors, including significantly decreasing the frequency of sex with two or more partners, oral and anal sex, and condomless anal sex from February to April 2020. However, these behaviors significantly increased from April to June 2020. Conversely, a recent cross-sectional survey of gay, bisexual, and other MSM found that participants, in general, did not report reducing the number of sex partners during the onset of the pandemic, and in fact had significantly increased the number of sex partners, anal sex partners, and a marginal increase in the number of condomless anal sex partners during the onset of the pandemic (February to April 2020) [10]. These increases in sexual behavior were associated with increased substance use during this time period [10].

Structural-level factors, including changes in clinical hours and/or conversion from in-person PrEP visits to telehealth visits, may also impede and/or enhance PrEP care engagement depending on access to technology. For instance, many PrEP and HIV care providers reported dramatic increases in telehealth visits during the onset of the COVID-19 pandemic, however, the extent to which telehealth services for PrEP and HIV care are accessible to all patients, including vulnerable and underserved patients, is relatively unknown $[1,3,7,8]$. Additionally, in order to reduce structural barriers even some pharmacists and pharmacies have considered or are engaged in providing PrEP care to pharmacy clients as PrEP care becomes increasingly limited during the pandemic $[1,11]$.

At the onset of the COVID-19 pandemic many state and county health departments and HIV/STI clinics were called upon to reallocate efforts of HIV and STI epidemiologists, disease tracers, and outreach, education, and prevention experts to the local COVID-19 response, which may have direct impact on access to HIV and STI prevention and care. In fact, the National Association of County and City Health Officials (NACCHO) reported in early April that of the over 3000 county and city health departments surveyed, many reported reduced or suspended HIV and STI services and prevention activities, with several reporting they were no longer starting clients on PrEP [12]. Further, many local health authorities were only prioritizing cases of symptomatic or confirmed STIs and the contacts of people who had tested positive, minimizing tracing. Co-managing the pandemic response effort, in addition to already increasing STI rates [13], is likely to overburden the local public health infrastructure, suggesting alternative health care facilities, including community-based programs and independent sexual and reproductive health clinics, may play a critical role in providing HIV prevention care during the pandemic.

The purpose of this study was to explore the number of pre-exposure prophylaxis (PrEP), HIV testing, and STI testing visits that occurred during the onset of the COVID-19 pandemic among patients attending eight sexual and reproductive health clinics located in three HIV priority states in the Ending the HIV Epidemic (EHE): A Plan for America: Arkansas, Missouri, and Oklahoma.

\section{Methods}

Patient visit type and prescription data was extracted from the electronic health record system (EHR) [NextGen ${ }^{\circledR}$ Healthcare: Irvine, CA] of a multi-state non-profit network of sexual and reproductive health care clinics providing care in Arkansas, Missouri, and Oklahoma, with a focus on male patients who were receiving PrEP care and all patients receiving HIV and STI testing between March 1, 2019 and June 30, 2019 (pre-COVID-19 pandemic) and March 1, 2020 and June 30, 2020 (early onset of the COVID-19 pandemic). Our PrEP analyses focus on male patients as the majority of PrEP-using patients were assigned male at birth. PrEP-using patients who were assigned female at birth represented only $15.3 \%(n=15)$ and $13 \%(n=12)$ of the total number of PrEP visits in 2019 and 2020, respectively. Given the small number of female PrEP visits, analyses based on assigned sex at birth and month did not meet statistical assumptions, with several cells having less than five patients for comparisons. Data was tabulated using Tableau ${ }^{\circledR}$ Data Software (Seattle, WA). We defined a PrEP visit as receiving a new or refill prescription visit (Truvada ${ }^{\circledR}$ or Descovy ${ }^{\circledR}$ ) and/or follow-up PrEP counseling/testing in-person or via telehealth/telePrEP visit; HIV testing visit as receiving in-person HIV testing and counseling; and STI testing visit as receiving in-person STI testing for chlamydia, gonorrhea, and/or syphilis. Telehealth for HIV and STI testing was not available during 
either the pre-pandemic or onset COVID-19 pandemic periods. All telehealth visits for PrEP were provisional and part of an emergency protocol allowing for medical history to determine PrEP eligibility for new patients. Existing patients were allowed to continue without intermediate HIV, STI, and renal function testing. Subsequent in-person HIV, STI, and renal function testing are necessary for long-term PrEP use.

Descriptive statistics were used to describe the frequency of PrEP visits, HIV testing visits, and STI testing visits in total and for each month in 2019 and 2020 (March-June). Additionally, proportions for the number of male patient visits that were PrEP visits and proportions of all patient HIV and STI testing visits were calculated for both time periods. Next, two-sample $z$-tests of proportions were used to calculate differences in the proportion of male PrEP visits and the proportions of all patient HIV testing visits and STI testing visits year-over-year (2019/2020) by month (March-June), and in total during the initial four month period of the COVID-19 pandemic. Comparison analyses were conducted using $R$ Statistical Software (Vienna, Austria).

\section{Results}

Overall, a total of 80 male PrEP visits were provided during the first four months of the COVID-19 pandemic (March 1, 2020-June 30, 2020) across eight sexual and reproductive health clinics in Arkansas, Missouri, and Oklahoma. Thirteen patient visits (16.3\%) were "new" PrEP patients, with 47 (58.8\%) being "established" PrEP patients and 20 (24.9\%) being "unknown". The vast majority of patients receiving PrEP care during the pandemic were using or prescribed Truvada ${ }^{\circledR}$ for PrEP $(81.2 \%)$, with $18.8 \%$ using or prescribed Descovy ${ }^{\circledR}$. Among 83 PrEP visits provided from March 1, 2019 to June 30, 2019, 26 (31.3\%) were "new" PrEP patients, 37 (44.6\%) were "established" PrEP patients, and $20(24.1 \%)$ were "unknown". A greater proportion of PrEP visits in 2019 were among new patients, compared to $2020(z=2.07, p=0.04, d=0.36)$. There were no significant differences observed for the proportions of established $(z=-1.65, p=0.10, d=0.28)$ or unknown $(z=0$, $p=1.0, d=0.02$ ) patients between 2019 and 2020. In 2019 all patients were using or prescribed Truvada ${ }^{\circledR}$ for PrEP. For both time periods, the majority of PrEP visits were provided in Oklahoma (2020: 51.3\%; 2019: 48.2\%), followed by Missouri (31.2\%; 36.1\%), and Arkansas (17.5\%; 15.7\%). All PrEP visits provided in 2019 were provided in-person in the clinic. However, 59 (73.8\%) PrEP visits provided during the onset of the COVID-19 pandemic were provided via telehealth/telePrEP, telephone and/or video conferencing with a clinician, with the majority $(69.2 \%)$ provided to "established" patients.

Table 1. details the total number of male patient visits, the proportion of male visits that were PrEP visits, total HIV and STI testing visits, and total sexual and reproductive health patient visits. Comparison tests revealed that a significantly greater proportion of male PrEP visits occurred during the first four months of the COVID-19 pandemic, compared to male PrEP visits during the same time period in the previous

Table 1 PrEP, HIV, and STI visits among patients receving care prior to and during the onset of the COVID-19 pandemic

\begin{tabular}{|c|c|c|c|c|c|c|c|}
\hline & 2019 & & 2020 & & $z$ & $p$ & $d$ \\
\hline & Total male visits & Male PrEP visits (\%) & Total male visits & Male PrEP visits (\%) & & & \\
\hline March & 435 & $18(4.1)$ & 280 & $25(8.9)$ & -2.47 & 0.014 & 0.20 \\
\hline April & 436 & $22(5.1)$ & 168 & $19(11.3)$ & -2.56 & 0.01 & 0.23 \\
\hline May & 424 & $28(6.6)$ & 223 & $14(6.3)$ & 0 & 1.0 & 0.01 \\
\hline June & 413 & $15(3.6)$ & 246 & $22(8.9)$ & -2.69 & 0.007 & 0.22 \\
\hline Total & 1708 & $83(4.9)$ & 917 & $80(8.7)$ & -3.83 & $<0.001$ & 0.16 \\
\hline & Total patient visits & Total HIV testing visits (\%) & Total patient visits & Total HIV testing visits (\%) & & & \\
\hline March & 2933 & $615(21.0)$ & 2279 & $343(15.1)$ & 5.44 & $<0.001$ & 0.15 \\
\hline April & 3122 & $586(18.8)$ & 1635 & $201(12.3)$ & 5.67 & $<0.001$ & 0.18 \\
\hline May & 3099 & $622(20.1)$ & 1777 & $175(9.8)$ & 9.25 & $<0.001$ & 0.29 \\
\hline June & 2948 & $629(21.3)$ & 2226 & $294(13.2)$ & 7.52 & $<0.001$ & 0.22 \\
\hline Total & 12,102 & $2452(20.3)$ & 7917 & $1013(12.8)$ & 13.63 & $<0.001$ & 0.20 \\
\hline & Total patient visits & Total STI testing visits (\%) & Total patient visits & Total STI testing visits (\%) & & & \\
\hline March & 2933 & $2098(71.5)$ & 2279 & $1080(47.4)$ & 17.69 & $<0.001$ & 0.50 \\
\hline April & 3122 & $2031(65.1)$ & 1635 & $658(40.2)$ & 16.36 & $<0.001$ & 0.50 \\
\hline May & 3099 & $1963(63.3)$ & 1777 & $554(31.2)$ & 21.60 & $<0.001$ & 0.66 \\
\hline June & 2948 & $1998(67.8)$ & 2226 & $875(39.3)$ & 20.37 & $<0.001$ & 0.58 \\
\hline Total & 12,102 & $8090(66.8)$ & 7917 & $3167(40.0)$ & 37.42 & $<0.001$ & 0.55 \\
\hline
\end{tabular}


year $(z=-3.83, p<0.001)$. This difference had a small effect size $(d=0.16)$. Monthly year-over-year comparisons of male PrEP visits suggest a significantly greater proportion of male visits were used for PrEP for every month during the onset of the pandemic with the exception of May $(z=0$, $p=1.0, d=0.01$ ).

A significantly smaller proportion of HIV testing visits were provided during the COVID-19 pandemic period, with an overall $58.7 \%$ decrease in HIV tests provided compared to the pre-pandemic period $(z=13.63, p<0.001)$. This difference had a small effect size $(d=0.20)$. Similar to HIV testing visits, a significantly smaller proportion of STI testing visits were provided during the COVID-19 pandemic period, with an overall $60.9 \%$ decrease in STI tests provided compared to the pre-pandemic period $(z=37.42, p<0.001)$. This difference had a medium effect size $(d=0.55)$.

\section{Discussion}

Overall, our findings suggest that PrEP care remained a priority for male patients in Arkansas, Missouri, and Oklahoma during the onset of the COVID-19 pandemic. In fact, although clinic hours were reduced during this time in order to comply with social distancing practices and shelter-inplace orders, when appointments were available, PrEP care visits were consistent with pre-pandemic PrEP care visits. This finding is particularly notable given other studies have reported interruptions to PrEP services, including decreased access to and usage of PrEP, during the COVID-19 pandemic $[6-10,14]$. The larger proportion of male PrEP visits is likely at least partially attributable to the availability of PrEP appointments via telehealth, as a majority (73.8\%) were delivered through this technology. For existing patients, the increased availability of telehealth PrEP visits allowed for continuity of care without requiring in-person visits even during the onset of the pandemic. Although telehealth PrEP care provides patient continuity and a remote way to access PrEP, this care does not fully replicate an inperson PrEP visit. Innovations and systems improvements in at-home HIV and STI testing as well as CDC recommended renal function testing are needed to comprehensively provide PrEP care via telehealth/telePrEP, without subsequent inperson testing visits [15]. In addition, because HIV prevention efforts at many local health authorities have been scaled back or suspended due to the COVID-19 pandemic response [10], patients may be relying on other healthcare organizations for continued PrEP access. For those patients who ordinarily rely on HIV prevention care through local health authorities, ensuring that care is accessible through other sexual and reproductive health clinics, particularly via telehealth, may be especially crucial under current conditions.
Generally, there were significantly fewer in-person HIV and STI testing visits provided during the onset of the COVID-19 pandemic. Even with diminished numbers of testing visits overall, over 3000 STI testing visits and 1000 HIV testing visits were provided during the onset of the pandemic period. These findings demonstrate relatively consistent engagement with STI and HIV prevention among our patient population. However, given the high volume of HIV and STI testing at these clinics overall and especially among the female patient population, there may be a missed opportunity to counsel and engage all patients receiving HIV and STI testing with PrEP for HIV prevention. Additionally, given that currently HIV and STI testing visits are only given in-person, innovations including at-home testing may increase testing rates by eliminating the need for solely inperson visits.

\section{Limitations}

Our findings offer promising insights into the utilization of PrEP care and HIV and STI testing visits in three EHE priority states, however there are limitations to our study. First, this study relies on system-level data and does not include individual patient-level variables, including sexual risk behaviors, PrEP adherence, adequate measures of Truvada ${ }^{\circledR}$ or Descovy ${ }^{\circledR}$ to offer protection from HIV, individual barriers or facilitators to accessing, initiating, and/or maintaining PrEP or testing services during a pandemic, and/ or broader social determinants of health that may impact access to care or increased risk for COVID-19 infection, in addition to STIs and HIV. Subsequent studies focused on individual PrEP users and their experiences seeking and maintaining PrEP care, as well as those seeking HIV and STI testing during the COVID-19 pandemic are needed to inform individual-level support programs and interventions. Second, our analyses focus on the earliest months of the pandemic based on the early cases of COVID-19 in the Midwest and South. However, these dates may not adequately reflect the steepest increase in COVID-19 infection and deaths in this geography, as only in more recent months has the Midwest and South seen a steep increase in COVID-19 infection. The potential relationship between shelter-in-place and the spiking incidence in COVID-19 infection are beyond the scope of this paper and our dataset, however there are likely important associations between these factors and the use of both in-person HIV and STI testing as well as PrEP care, whether in-person or through telehealth/telePrEP. Lastly, given the small sample size of male PrEP visits in total we were unable to explore variability based on new and established PrEP patients, recognizing there may be important individual contextual factors that would inform a patient's decision to initiate PrEP during the COVID-19 pandemic 
that are likely to be different from PrEP use prior to the pandemic.

\section{Conclusion}

We found during the initial four months of the COVID-19 pandemic, male PrEP visits remained relatively consistent with pre-pandemic PrEP care across eight sexual and reproductive health clinics in three Ending the HIV Epidemic (EHE) high priority states. The majority of these visits were provided via telehealth/telePrEP. These findings suggest when telehealth visits for PrEP care remained available to male patients during the pandemic, patients continued to utilize these services. Telehealth/telePrEP in particular appears to be a promising avenue for PrEP care administration during the COVID-19 pandemic. However, overall we found fewer in-person HIV and STI testing visits were provided during the COVID-19 pandemic period, compared to the pre-pandemic period. Decrease in in-person visit availability due to social distancing may impede patient access to care, particularly for walk-in services like HIV and STI testing. Thus, there may be an opportunity for potential innovations in increased access to in-home HIV and STI testing. Further, in order to continue making improvements in this area, community-based health centers, including independent non-profit sexual and reproductive health clinics, may fill a critical gap while health departments in these states prioritize mitigating the impact of COVID-19. These combined efforts may be essential in staying on track to achieve the proposed EHE goals of reducing HIV incidences in disproportionately impacted geographical areas of the US, particularly the Southern US.

Acknowledgements We would like to acknowledge and thank all the clinicians, health care providers, and health services staff who continue to provide critical sexual and reproductive health care every day across the Planned Parenthood Great Plains network of health centers. The authors of this publication did not receive any direct funding for this publication.

\section{Compliance with Ethical Standards}

Conflict of interest The authors have no conflict of interest to declare.

Ethical approval All protocols and procedures performed in this study were deemed exempt by the organization's Institutional Review Board.

\section{References}

1. Shoptaw S, Goodman-Meza D, Landovitz RJ. Collective call to action for HIV/AIDS community-based collaborative science in the era of COVID-19. AIDS Behav. 2020;24:2013-6.

2. Pinto RM, Park S. COVID-19 pandemic disrupts HIV Continuum of care and prevention: implications for research and practice concerning community-based organizations and frontline providers. AIDS Behav. 2020;24(9):2486-9.

3. Ridgway JP, Schmitt J, Friedman E, Taylor M, Devlin S, McNulty M, Pitrak D. HIV care continuum and COVID-19 outcomes among people living with HIV during the COVID-19 pandemic, Chicago IL. AIDS Behav. 2020;24:2770-2.

4. Fauci AS, Redfield RR, Sigounas G, Weahkee MD, Giroir BP. Ending the HIV epidemic: a plan for the United States. JAMA. 2019;321(9):844-5.

5. United States Health and Human Services. Ending the HIV epidemic: a plan for America. 2019 [updated February 9, 2019]. https://www.hhs.gov/blog/2019/02/05/ending-the-hiv-epidemica-plan-for-america.html. Accessed 4 Aug 2020.

6. Chow EPF, Hocking JS, Ong JJ, Schmidt T, Buchanan A, Rodriguez E, Maddaford K, Patel P, Fairley CK. Changing the use of HIV pre-exposure prophylaxis among men who have sex with men during the COVID-19 pandemic in Melbourne. Aust Open Forum Infect Dis. 2020;7(7):1-4.

7. Brawley S, Dinger J, Nguyen C, Anderson J. Impact of COVID19-related shelter-in-place orders on PrEP access, usage and HIV risk behaviors in the United States. In: Paper presented at International AIDS Conference; July 6-10, 2020 (virtual meeting).

8. Krakower D, Solleveld P, Levine K, Mayer KH. Impact of COVID-19 on HIV pre-exposure prophylaxis care at a Boston community health center. In: Paper presented at International AIDS Conference; July 6-10, 2020 (virtual meeting).

9. Pampati S, Emrick K, Siegler AJ, Jones J. Changes in sexual behavior and access to sexual health services due to the COVID19 pandemic among PrEP-using MSM in the South. In: Presented at STD Prevention Conference; September 14-24, 2020 (virtual meeting).

10. Stephenson R, Chavanduka TMD, Rosso MT, Sullivan SP, Pitter R, Hunter AS, Rogers E. Sex in the time of COVID-19: results from an online survey of gay, bisexual and other men who have sex with men's experience of sex and HIV prevention during the US COVID-19 epidemic. AIDS Behav. 2020. https://doi. org/10.1007/s10461-020-03024-8.

11. Crawford ND, Albarran T, Chamberlain A, Hopkins R, Josma D, Morris J, et al. Willingness to discuss and screen for pre-exposure prophylaxis in pharmacies among men who have sex with men. $\mathrm{J}$ Pharm Pract. 2020. https://doi.org/10.1177/0897190020904590.

12. Kelly K. LHD HIV, STI, and hepatitis programs respond and adapt to COVID-19 (April 10, 2020). National Association of County and City Health Officials (NACCHO). https://www.naccho.org/ blog/articles/lhd-hiv-sti-and-hepatitis-programs-respond-andadapt-to-covid-19. Accessed 4 Aug 2020.

13. Centers for Disease Control and Prevention. Sexually transmitted disease surveillance 2018 [updated July 30, 2019]. https://www. cdc.gov/std/stats18/default.htm. Accessed 4 Aug 2020.

14. Sanchez TH, Zlotorzynska M, Rai M, Baral SD. Characterizing the impact of COVID-19 on men who have sex with men across the United States in April, 2020. AIDS Behav. 2020;24(7):2024-32.

15. Armstrong WS, Agwu AL, Barrette EP, Ignacio RB, Chang JJ, Colasanti JA, Floris-Moore M, Haddad M, MacLaren L, Weddle A. Innovations in human immunodeficiency virus (HIV) care delivery during the coronavirus disease 2019(COVID-19) pandemic: policies to strengthen the ending the epidemic initiative-a policy paper of the infections disease society of American and the HIV medicine association. Clin Infect Dis. 2020. https://doi. org/10.1093/cid/ciaa1532.

Publisher's Note Springer Nature remains neutral with regard to jurisdictional claims in published maps and institutional affiliations. 